\title{
Efeito do abacate (Persea americana Mill) variedade hass na lipidemia de ratos hipercolesterolêmicos
}

\author{
Effect of the hass avocado (American Persea Mill) on hipercolesterolemic rats
}

\author{
Jocelem Mastrodi SALGADO ${ }^{1 \star}$, Camila BIN ${ }^{1}$, Débora Niero MANSI ${ }^{1}$, Adriana SOUZA²
}

\begin{abstract}
Resumo
Os fitonutrientes podem ser encontrados em muitas frutas, sendo o abacate o mais rico em beta-sitosterol (fitoesterol) e glutationa. O fitoesterol é uma substância de origem vegetal cuja estrutura é muito similar a do colesterol e seu mecanismo de ação envolve a inibição intestinal de absorção do colesterol e diminuição da síntese de colesterol hepático. O efeito é exercido sobre os valores de colesterol plasmático total e LDL, sem afetar os níveis de HDL e triglicérides sanguíneos. O presente trabalho teve como objetivo analisar a influência do consumo de farinha de abacate variedade Hass nos níveis séricos de colesterol total, HDL, LDL, triglicérides, colesterol hepático e excretado. Estes parâmetros foram investigados em ratos Wistar hipercolesterolêmicos durante 30 e 60 dias de experimento. Ao final de 30 dias, a dieta com 15\% de abacate mostrou ser mais efetiva para reduzir os níveis de colesterol total e LDL e aumentar o HDL em comparação ao controle. Foi observado que para o teor de colesterol excretado a melhor dieta foi a de $25 \%$ de abacate, pois quanto maior a concentração de abacate, maior a excreção de colesterol. A dieta com $15 \%$ e $25 \%$ de abacate, foram as que mais influenciaram nos níveis de colesterol hepático.

Palavras-chave: abacate; colesterol; fitoesterol; doenças cardiovasculares.
\end{abstract}

\begin{abstract}
Phytonutrients can be found in fruits, and the avocado contains four times more beta-sitosterol (phytosterol) and it is one of the best glutathione sources. The phytosterol is a vegetable substance whose structure is very similar to the cholesterol one and its action mechanism involves the intestinal inhibition of cholesterol absorption and the synthesis of hepatic cholesterol. The effect has an influence on the values of total plasmatic cholesterol and LDL without affecting the levels of HDL and triglycerides. The present work had the objective to analyze the influence of the consumption of the Hass avocado on the levels of total cholesterol, HDL, LDL, triglycerides, and hepatic and excreted cholesterol. These parameters were investigated in hipercholesterolemic Wistar rats during 30 and 60 days of experiment. At the end of 30 days, the $15 \%$ avocado diet reduced the levels of total cholesterol and LDL in comparison to the control. It was observed that for the excreted cholesterol, the best diet was the $25 \%$ of avocado since the cholesterol excretion increased with the increase of the avocado concentration. The $15 \%$ avocado diet also influenced the levels of hepatic cholesterol.

Keywords: avocado; cholesterol; phytosterol; cardiovascular diseases.
\end{abstract}

\section{Introdução}

O panorama nacional das doenças cardiovasculares prevê uma verdadeira epidemia com conseqüências para a saúde pública, devido à tendência de elevação na sua mortalidade (SANTOS, 2001).

O controle de doenças cardiovasculares, em pacientes com hipercolesterolemia, pode ser feito com o auxílio de medicamentos, programas de atividade física e através da terapia nutricional. A alimentação é extremamente importante e deve sempre ser considerada ao se determinar o risco para as doenças cardiovasculares (MAZUR; DUKE; WAHALA, 1998; O'KEEFE; NELSON; HARRIS, 1996).

Estudos mostram que o consumo de dietas ricas em gorduras monoinsaturadas (ácido oléico), em substituição a gorduras saturadas, exerce seletivos efeitos fisiológicos sobre humanos, reduzindo os níveis de colesterol total, de triglicerídios e de LDL-colesterol, sem alterar a fração HDL-colesterol do plasma (REBOLLO et al., 1998; TURATTI et al., 1985).
A concentração de colesterol no plasma pode ser diminuída pela constante ingestão de ácidos graxos polinsaturados e redução de ácidos graxos saturados da dieta (MAGALHÃES; CHAGAS; Da LUZ, 2002).

Estudo de Hu (2003) observou efeitos benéficos do consumo de gordura monoinsaturada de origem vegetal como óleos de oliva, canola, nozes e abacate, com uma significante redução do risco de desenvolver doenças cardiovasculares.

O abacate é uma das frutas que mais se destaca pela sua qualidade nutricional. É rico em ácido oléico e $\beta$-sitosterol, uma gordura insaturada utilizada como coadjuvante no tratamento de hiperlipidemias. Em um estudo com dieta enriquecida com $1,68 \mathrm{~g}$ de fitosteróis por dia, observou-se a redução da concentração de colesterol total e o LDL em 10 e 12\%, respectivamente (LOTTENBERG, 2002).

Recebido para publicação em 25/7/2007

Aceito para publicação em 2/11/2007 (002705)

${ }^{1}$ Escola Superior de Agricultura Luiz de Queiroz - ESALQ, Universidade de São Paulo - USP, CEP13418900, Piracicaba - SP, Brasil, E-mail: jmsalgad@esalq.usp.br

2 Centro de Energia Nuclear na Agricultura - CENA, Universidade de São Paulo - USP, Piracicaba - SP, Brasil

${ }^{*}$ A quem a correspondência deve ser enviada 
O fitoesterol é uma substância de origem vegetal cuja estrutura é muito similar a do colesterol. São descritas numerosas propriedades fisiológicas para os fitoestérois, sendo o efeito hipocolesterolêmico o melhor caracterizado e que se observa em indivíduos alvo de moderada hipercolesterolemia (DUESTER, 2001). O mecanismo de efeito do fitoesterol envolve a inibição intestinal de absorção do colesterol e diminuição da síntese de colesterol hepático (MOGHANDASIAN; FROHLICH, 1999). O efeito se exerce sobre os valores de colesterol plasmático total e LDL, sem afetar os níveis de HDL e triglicerídios sanguíneos (VALENZUELA; GARRIDO, 2000). Uma dieta balanceada fornece de 200 a $400 \mathrm{mg}$ de fitosteróis, mas a ingestão de 3 a $4 \mathrm{~g} /$ dia destes promove a redução do LDL colesterol em 10 a 15\% (SANTOS et al., 2001). Na maioria das vezes, na substituição de gorduras saturadas por insaturadas, as concentrações de colesterol são reduzidas e as de HDL aumentadas (RIQUE; SOARES; MEIRELLES, 2002).

Tendo em vista o potencial efeito benéfico do abacate, o objetivo deste trabalho foi analisar a influência do consumo de farinha de abacate variedade Hass nos níveis séricos de colesterol total, HDL, LDL, triglicérides, colesterol hepático e excretado.

\section{Material e métodos}

\subsection{Matéria-prima}

Foram utilizados abacates maduros da variedade Hass obtidos no comércio local de Piracicaba. Os abacates foram higienizados com solução de água clorada (100 ppm), cortados em pedaços e colocados em bandejas para secagem em estufa com circulação de ar forçada, em temperatura entre 55 e $60{ }^{\circ} \mathrm{C}$, durante 48 horas. Uma solução de ácido cítrico 1\% foi distribuída sobre cada bandeja, para evitar o escurecimento enzimático. As amostras secas foram moídas em moinho de facas, a farinha obtida foi conservada em sacos de polietileno transparentes e mantida sob refrigeração $\left(4^{\circ} \mathrm{C}\right)$ até o momento do uso.

\subsection{Análises químicas do abacate}

Os teores de umidade, proteína, lipídios e cinza foram determinados de acordo com a metodologia descrita pela AOAC (1995). O teor de fibras solúveis e insolúveis foi avaliado pelo método proposto por ASP et al. (1983).

\subsection{Ensaio biológico}

\section{Preparo das dietas}

As dietas foram elaboradas de acordo com o American Institute of Nutrition (REEVES; NIELSEN; FAHEY, 1993), com adição de banha de porco, contendo $206 \mathrm{mg} .100 \mathrm{~g}^{-1}$ de colesterol (PREGNOLATTO; PREGNOLATTO, 1985). As dietas utilizadas para o ensaio biológico foram: controle (15\% caseína), 5, 15 e 25\% de farinha de abacate (FA) e encontram-se na Tabela 1.

\section{Animais}

Foram utilizados ratos adultos (Rattus norvegicus var. albinos), machos, da linhagem Wistar, com idade média de 3 a 4 meses, pesando entre 230 e 250 g. No período pré- experimental, os animais foram mantidos em gaiolas coletivas ( 6 animais por gaiola), em temperatura controlada $\left(22\right.$ a $23{ }^{\circ} \mathrm{C}$ ), com ciclo de claro-escuro de 12 horas, recebendo ração comercial Purina e água ad libitum até atingirem peso ideal.

Foram selecionados 54 ratos que consumiram dieta hipercolesterolêmica durante uma semana. Destes, 6 ratos (Tempo 0 - T0) foram sacrificados após esta semana a fim de serem utilizados como parâmetro de comparação, e foram dosados os níveis sanguíneos de colesterol total, LDL-C, HDL-C e triglicerídeos.

Os 48 animais restantes foram divididos em 4 grupos: controle (dieta caseína), 5, 15 e 25\% de FA (Dieta enriquecida com diferentes porcentagens de Farinha de Abacate), formados por 12 animais cada. Para cada dieta, 6 animais foram sacrificados aos 30 dias e os outros 6 aos 60 dias de experimento. Esses animais permaneceram em gaiolas individuais, em ambiente com temperatura controlada de 22 e $23^{\circ} \mathrm{C}$, iluminação ambiente controlada para 12 horas de luz e 12 horas de obscuridade, recebendo água e alimento ad libitum, sendo o registro do ganho de peso e do consumo de dieta realizados 3 dias por semana.

Durante todo o período experimental, 3 vezes por semana, as fezes dos animais dos grupos controle inicial, controle, 5, 15 e 25\% FA, em ambos os tempos, foram coletadas em sacos plásticos individuais, identificados e armazenados no refrigerador. No final de todo o experimento, as fezes foram secas em estufa a $105^{\circ} \mathrm{C}$ por 72 horas e moídas para análise.

No final de cada período, os animais foram sacrificados, após jejum de 12 horas, para coleta de sangue e fígado. As amostras foram analisadas logo após a coleta.

\section{Análises bioquímicas}

Os animais foram submetidos ao sacrifício por processo anestésico com halotano e o sangue foi coletado através de punção cardíaca. Para as análises de colesterol total, HDL-C e triglicerídios, utilizou-se o Kit da Wiener Lab. Os teores de LDL-C foram identificados pela Equação 1 (FRIEDEWALD; LEVY; FREDRICKSON, 1972).

Tabela 1. Composição das dietas $\left(\mathrm{g} .100 \mathrm{~g}^{-1}\right)$ controle e suplementadas com farinha de abacate variedade Hass.

\begin{tabular}{lcccc}
\hline \multicolumn{1}{c}{ Componentes } & \multicolumn{4}{c}{ Dietas } \\
\cline { 2 - 5 } & Controle & $5 \% \mathrm{FA}$ & $15 \% \mathrm{FA}$ & $25 \% \mathrm{FA}$ \\
\hline Caseína $^{1}$ & 15,0 & 15,0 & 15,0 & 15,0 \\
Farinha de abacate $^{2}$ & - & 5,0 & 15,0 & 25,0 \\
Mistura mineral $^{3}$ & 3,5 & 3,5 & 3,5 & 3,5 \\
Mistura vitamínica $^{4}$ & 1,0 & 1,0 & 1,0 & 1,0 \\
Óleo de soja $^{5}$ & 3,0 & 3,0 & 3,0 & 3,0 \\
Banha de porco $^{6}$ & 7,0 & 7,0 & 7,0 & 7,0 \\
Bitartarato de colina $^{7}$ & 0,25 & 0,25 & 0,25 & 0,25 \\
Sacarose $^{8}$ & 10,0 & 10,0 & 10,0 & 10,0 \\
$\alpha$ - cistina & 0,3 & 0,3 & 0,3 & 0,3 \\
Tert - butilhidroquinona $^{10}$ & 0,0014 & 0,0014 & 0,0014 & 0,0014 \\
Amido $^{11}$ & \multicolumn{5}{c}{ Para completar 100\% } \\
\hline
\end{tabular}

${ }^{1}$ Synth; ${ }^{2}$ Variedade Hass; ${ }^{3,4}$ Reeves et al. (1993); ${ }^{5}$ isa ${ }^{\infty} ;{ }^{6}$ Sadia $; ;{ }^{7}$ Synth; ${ }^{8}$ Vetec; ${ }^{9}$ Synth; ${ }^{10}$ Sigma; e ${ }^{11}$ Maizena ${ }^{\circ}$. 
$\mathrm{LDL}=$ Colesterol Total $-\mathrm{HDL}-\left(\frac{\text { Triglicerídio }}{5}\right)$

Após o sacrifício, o fígado dos animais foi retirado e preparado através do método proposto por Haug e Hostmark (1987) e, para a determinação do colesterol hepático, foi utilizado o mesmo kit descrito anteriormente.

A determinação de colesterol excretado foi feita conforme a análise realizada no fígado.

\subsection{Análise estatística}

Foi utilizado o programa estatístico SAS (1998), no qual foi aplicado o teste F para análise de variância e o teste de Tukey, para identificar diferenças estatísticas entre as médias. Foi estabelecido nível de significância $\mathrm{p} \leq 0,05$ para o teor de colesterol total, HDL-c, LDL-c, triglicerídio, colesterol hepático e excretado.

\section{Resultados e discussão}

\subsection{Composição centesimal}

Os resultados das análises da composição química do abacate variedade Hass, encontram-se na Tabela 2.

Pode-se observar que $100 \mathrm{~g}$ de abacate fresco contêm 3,13 g de Fibra Alimentar, representando $12 \%$ das necessidades diárias. Os valores de fibras encontrados estão de acordo com os citados por Duester (2001), no qual foram encontrados 3,4 g de fibras totais. Porém os valores de umidade e lipídios diferem dos encontrados, para a mesma variedade, por Tango et al. (2004) que, em estudo com diversas variedades de abacate, afirmam que os frutos que apresentaram teores baixos de umidade tiveram porcentagens de lipídios mais elevadas e vice-versa, assim, a alta correlação entre umidade e lipídios deve-se ao fato de que a soma dos teores dessas substâncias corresponde à grande parte da massa das polpas.

\subsection{Minerais}

A Tabela 3 mostra a composição mineral do abacate variedade Hass. Os valores encontrados são para os minerais: fósforo, potássio, cálcio, magnésio, enxofre, cobre, ferro, manganês, zinco e sódio.

A análise de minerais mostrou uma quantidade alta de ferro, potássio e zinco, ingredientes de grande valor para uma dieta balanceada.

Tabela 2. Composição química do abacate variedade Hass ( $\left.\mathrm{g} .100 \mathrm{~g}^{-1}\right)$ na matéria fresca.

\begin{tabular}{lc}
\hline Composição & Quantidade \\
\hline Umidade & 77,3 \\
Extrato etéreo & 10,8 \\
Proteína & 1,2 \\
Cinzas & 2,5 \\
Fibras insolúveis & 2,56 \\
Fibras solúveis & 0,57 \\
\hline
\end{tabular}

\subsection{Ensaio biológico}

\section{Colesterol}

A dieta que apresentou um maior efeito na redução dos níveis de colesterol foi a dieta com 15\% de FA (73,9 mg.dL $\left.\mathrm{dL}^{-1}\right)$, comparada ao nível inicial $\left(93,3 \mathrm{mg} \cdot \mathrm{dL}^{-1}\right)$, o que representou uma redução de $20,7 \%$ para os 30 dias de experimento. Houve uma redução mais acentuada dos valores após 60 dias de experimento como mostrado na Figura 1.

Independentemente do tempo de análise, a dieta que mais influenciou os níveis de colesterol total foi a dieta de $15 \%$ de FA, seguida pela dieta de $5 \%$.

A queda dos níveis de colesterol observada foi semelhante à encontrada por Valenzuela e Garrido (2000) que afirmam que uma dieta suplementada com esteróis vegetais reduz a hipercolesterolemia, possibilitando a prevenção, e ajuda no tratamento das doenças cardiovasculares. Em outro estudo, utilizando-se dieta enriquecida com 1,68 g de fitosteróis por dia, observou-se redução na concentração de colesterol total e LDL em 10 e 12\%, respectivamente (LOTTENBERG et al., 2002).

Hallikainen et al. (2000) avaliaram a ingestão de margarina com ésteres de fitostanóis ou fitosteróis, em pacientes hipercolesterolêmicos. Os pacientes consumiram durante 4 semanas fitostanóis ou fitosteróis, na dose de 2,01 a 2,04 g/dia. Houve redução significativa do colesterol total em 9,2 e 7,3\% e de LDL-c em 12,7 e 10,4\%, respectivamente, para estanóis e esteróis, na dose de 2,04 g/dia. Resultados semelhantes foram obtidos por Judd et al. (2002) que utilizaram, em molhos para salada, 3,6 g de fitosteróis e obtiveram diminuição no colesterol total de $7 \%$ e no LDL-c de $10 \%$, enquanto o HDL permaneceu inalterado. A diminuição de colesterol total e LDL-c com manutenção dos níveis de HDL têm intensa ligação com a diminuição de risco cardiovascular.

Segundo Lottenberg et al. (2002), a utilização de 3 g/dia de fitosteróis, na dieta em forma de margarina, por 60 adultos brasileiros, portadores de hipercolesterolemia moderada, promoveu redução de $10 \%$ do colesterol total e de $12 \%$ do LDL-c em um mês de intervenção. Resultados similares foram obtidos por Neil et al. (2001), com 32 pacientes a uma dose de 2,5 g/dia de fitosteróis, durante quatro meses.

Tabela 3. Composição mineral do abacate variedade Hass.

\begin{tabular}{lc}
\hline Minerais & Quantidades \\
\hline Fósforo $^{*}$ & 0,57 \\
Potássio* $^{*}$ & 18,21 \\
Cálcio $^{*}$ & 0,35 \\
Magnésio* $^{*}$ & 0,88 \\
Enxofre $^{*}$ & 0,67 \\
Cobre & 20,9 \\
Ferro $^{* *}$ & 19,7 \\
Manganês & 3,8 \\
Zinco & 27,3 \\
Sódio & 0,43 \\
\hline
\end{tabular}

${ }^{*}$ Valores expressos em g.kg-1 $\mathrm{e}^{* *}$ valores expressos em mg.kg-1. 
$H D L$

Para os níveis séricos de HDL, na Figura 2, não foram observadas diferenças estatisticamente significativas entre as dietas controle e 5\% de FA, entretanto, pode-se observar um aumento do nível de HDL para a dieta de 15\% de FA em 30 dias de experimento.

Aos 60 dias de experimento, os valores de HDL-c referentes às dietas de 5,15 e $25 \%$ se apresentaram iguais estatisticamente, porém, com a dieta de $15 \%$, os níveis de HDL foram $5 \%$ mais altos, em relação às dietas com 5 e $25 \%$ de FA.

Apesar dos valores de HDL diferirem estatisticamente entre as dietas 2 , 3 e 4, os teores de HDL são muito próximos, indicando não haver efeito da dieta, tanto para os 30 dias de experimento como para os 60 dias. O mecanismo de efeito do fitoesterol que envolve a inibição intestinal de absorção do colesterol, não afeta os níveis de HDL sanguíneo (VALENZUELA; GARRIDO, 2000), estando também de acordo com Hendriks (1999) que afirma que os valores se mantêm iguais devido ao mecanismo de absorção citado.

Colesterol - 30 dias

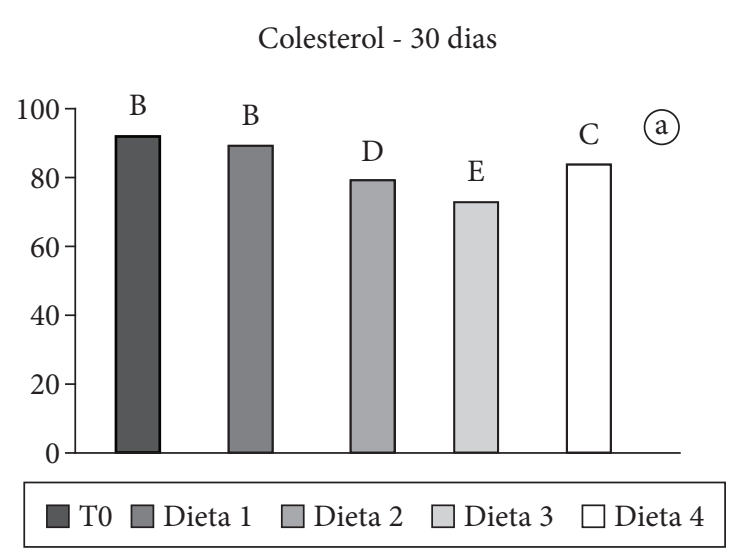

Entretanto, segundo McGowan (1997), as gorduras monoinsaturadas encontradas no azeite, óleo de canola, azeitonas, avelã, amêndoa e abacate são mais resistentes ao estresse oxidativo, e uma dieta rica nestes ácidos graxos faz com que as partículas de LDL-c fiquem enriquecidas com eles, tornando-as menos suscetíveis à oxidação (NORMÉN, 2000). Na substituição de gorduras saturadas por monoinsaturadas, as concentrações de colesterol total são reduzidas e as de HDL-c possivelmente aumentadas (McGOWAN, 1997).

\section{$L D L$}

Na Figura 3, são observadas diferenças estatísticas entre os valores de LDL nas dietas, indicando que a dieta de $15 \%$ de FA foi a que mais influenciou para a sua redução $\left(33,99 \mathrm{mg} \cdot \mathrm{dL}^{-1}\right)$ em 30 dias de experimento, observando-se resultados próximos para 60 dias de experimento.

A queda nos valores de LDL é influenciada pela dieta suplementada com esteróis vegetais, esse comportamento é esperado (HENDRIKS, 1999). Uma dieta balanceada, com quantidades de vegetais, fornece de 200 a 400 mg de fitosteróis, mas a ingestão de

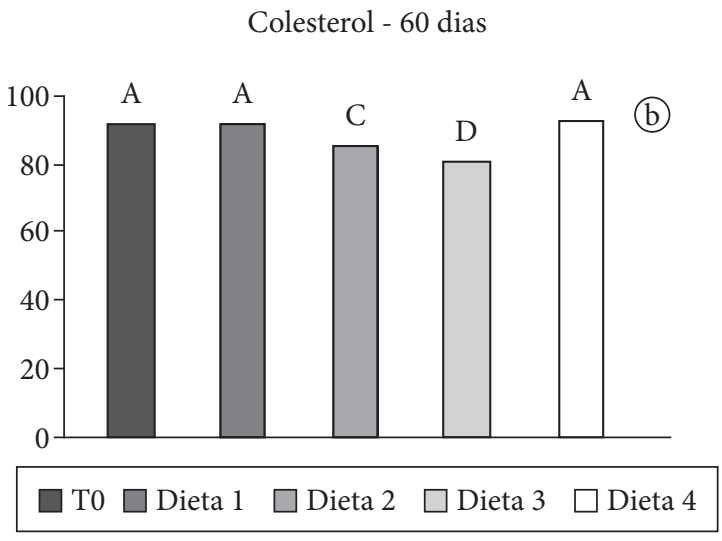

Figura 1. Níveis de Colesterol Sérico, em mg.dL ${ }^{-1}$, em ratos machos Wistar Hipercolesterolêmicos ${ }^{\star}$, obtidos no início, 30 e 60 dias de ensaio biológico. $\left(^{*}\right)$ valores médios de 6 animais, onde: T0 = valor inicial; dieta $1=$ dieta $15 \%$ caseína; dieta $2=5 \%$ de farinha de abacate; dieta $3=15 \%$ de farinha de abacate; dieta $4=25 \%$ de farinha de abacate; e as letras diferentes mostram diferença estatística significativa $(\mathrm{p}<0,05)$.
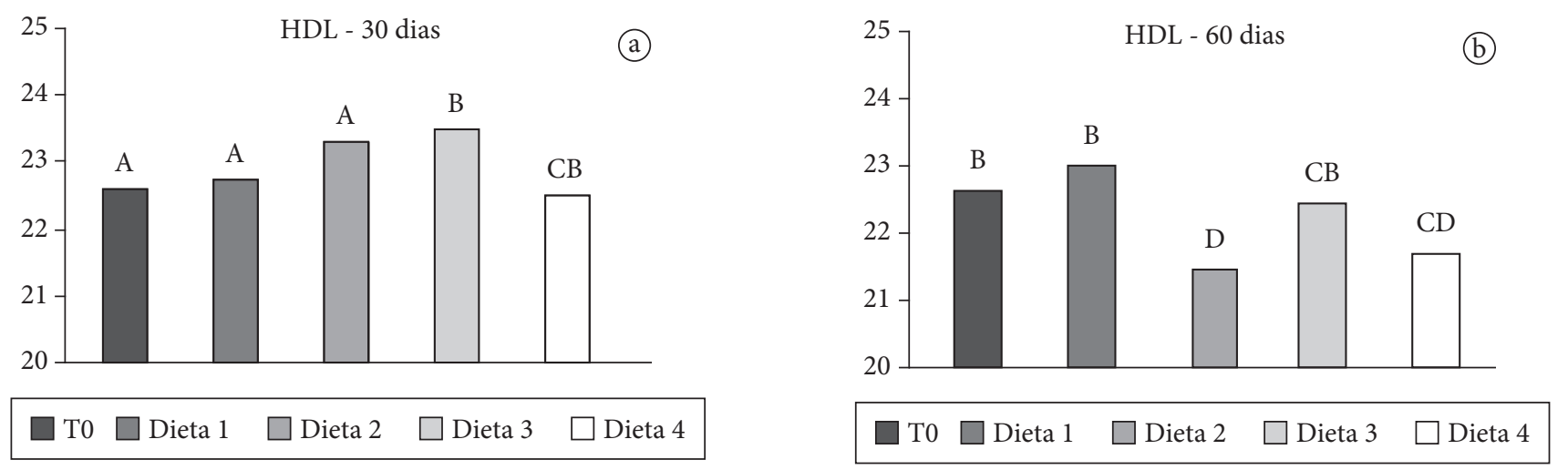

Figura 2. Níveis de HLD Sérico, em mg.dL ${ }^{-1}$, em ratos machos Wistar Hipercolesterolêmicos ${ }^{\star}$, obtidos no início, 30 e 60 dias de ensaio biológico. ${ }^{*}$ ) valores médios de 6 animais, onde: T0 = valor inicial; dieta $1=$ dieta $15 \%$ caseína; dieta $2=5 \%$ de farinha de abacate; dieta $3=15 \%$ de farinha de abacate; dieta $4=25 \%$ de farinha de abacate; e as letras diferentes mostram diferença estatística significativa $(\mathrm{p}<0,05)$. 
3 a 4 g/dia destes promove a redução do LDL colesterol em 10 a 15\% (RIQUE; SOARES; MEIRELLES, 2002). A redução da absorção de colesterol estimula o fígado a aumentar a síntese de colesterol, mas também aumenta o número de receptores LDL. Conseqüentemente, tanto os valores totais quanto os de LDL-colesterol diminuem, enquanto o valor de HDL-colesterol não se altera, permitindo assim, perfil lipídico adequado (HENDRIKS, 1999).

\section{Triglicerídios}

Na Figura 4, pode-se observar uma queda nos níveis de triglicerídios em todas as dietas testes, porém a dieta que mais influenciou na queda dos níveis de triglicerídios, em 30 dias de experimento, foi a dieta com 15\% de FA. Aos 60 dias de experimento foi observado um aumento nos valores de triglicerídios para todas as dietas, quando comparados aos resultados em 30 dias, e a dieta contendo $15 \%$ de farinha de abacate foi a que proporcionou uma maior redução.

A ausência de alteração significativa nos níveis de triglicerídios entre as dietas com 5 e $25 \%$ de farinha de abacate sugere que um aumento da proporção de farinha de abacate superior a $15 \%$ não mostrou benefícios quanto ao item estudado.

\section{Colesterol hepático}

Os menores valores de colesterol hepático foram encontrados para as dietas com 25\% (5,75 mg.dL $\left.{ }^{-1}\right)$ e $15 \%\left(6,09 \mathrm{mg} \cdot \mathrm{dL}^{-1}\right)$ de FA, aos 30 dias de experimento, e 15\% (7,94 mg.dL $\left.{ }^{-1}\right)$ aos 60 dias (Figura 5).

Os resultados do presente trabalho foram semelhantes aos encontrados por Naveh et al. (2002) que observaram um decréscimo significativo do colesterol hepático em ratos alimentados com dieta contendo polpa de abacate.

\section{Colesterol excretado}

Para ambos os tempos de análises, 30 e 60 dias, pode-se observar na Figura 6, que, quanto maior a concentração de farinha de abacate na dieta, maiores foram os valores de colesterol excretado.
LDL - 30 dias

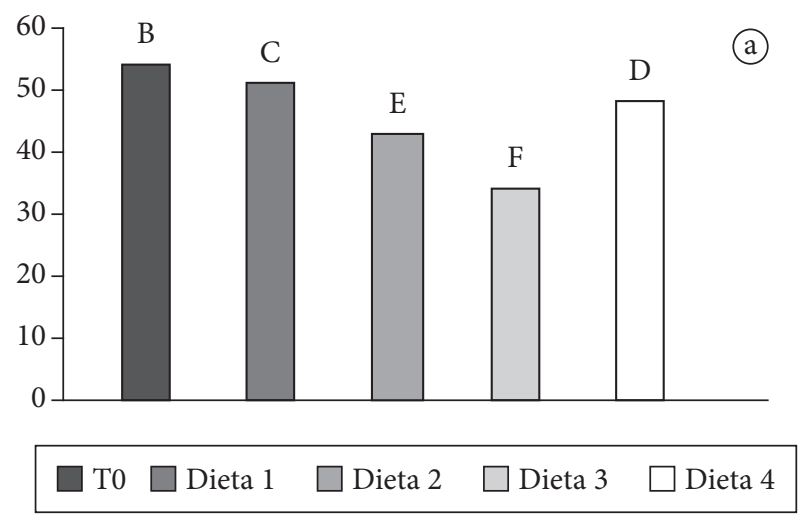

LDL - 60 dias

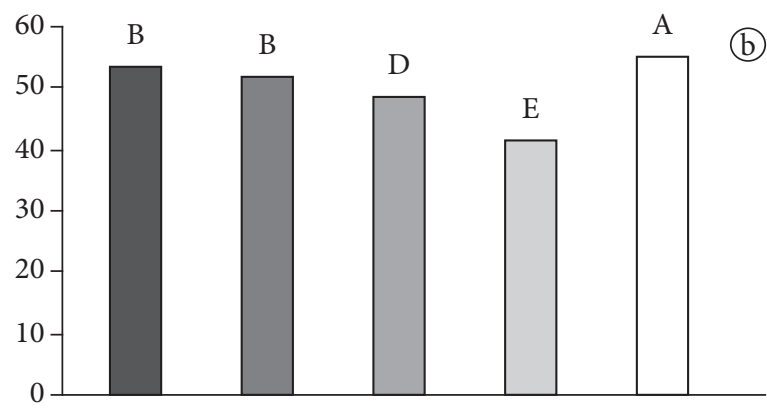

T0 $\square$ Dieta $1 \square$ Dieta $2 \square$ Dieta $3 \square$ Dieta 4

Figura 3. Níveis de LDL Sérico, em mg.dL $\mathrm{dL}^{-1}$, em ratos machos Wistar Hipercolesterolêmicos*, obtidos no início, 30 e 60 dias de ensaio biológico. ${ }^{*}$ ) valores médios de 6 animais, onde: T0 = valor inicial; dieta $1=$ dieta $15 \%$ caseína; dieta $2=5 \%$ de farinha de abacate; dieta $3=15 \%$ de farinha de abacate; dieta $4=25 \%$ de farinha de abacate; e as letras diferentes mostram diferença estatística significativa $(\mathrm{p}<0,05)$.
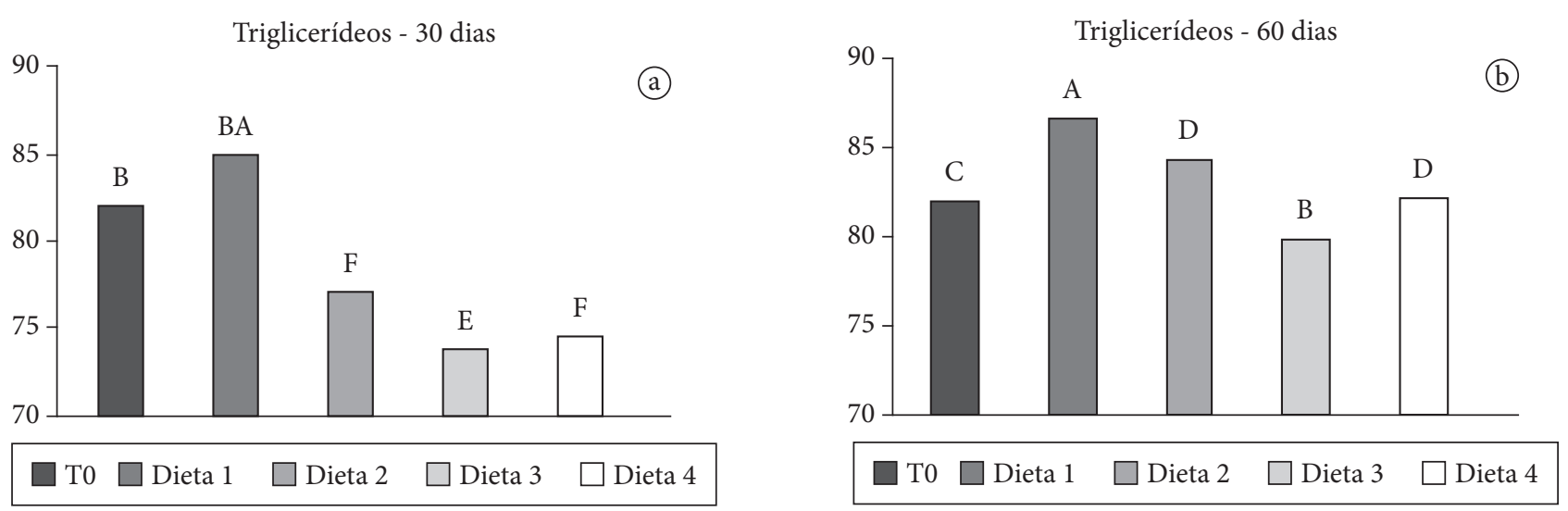

Figura 4. Níveis de Triglicérides Sérico, em mg.dL $\mathrm{d}^{-1}$, em ratos machos Wistar Hipercolesterolêmicos ${ }^{\star}$, obtidos no início, 30 e 60 dias de ensaio biológico. ${ }^{*}$ ) valores médios de 6 animais, onde: T0 = valor inicial; dieta $1=$ dieta $15 \%$ caseína; dieta $2=5 \%$ de farinha de abacate; dieta $3=15 \%$ de farinha de abacate; dieta $4=25 \%$ de farinha de abacate; e as letras diferentes mostram diferença estatística significativa $(\mathrm{p}<0,05)$. 

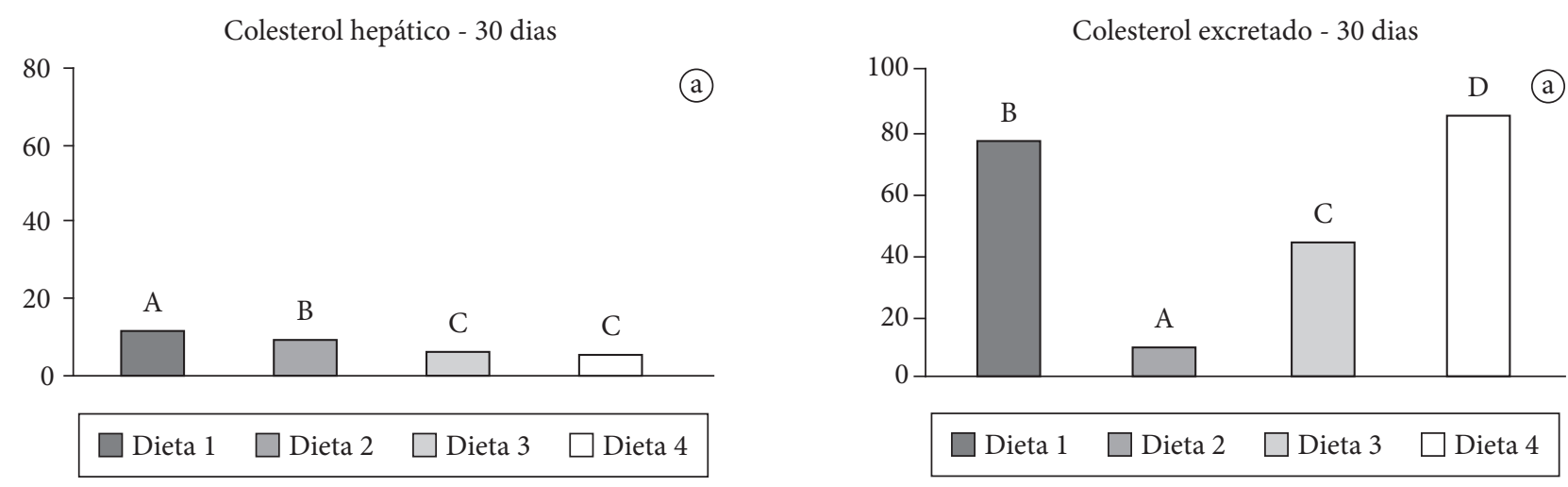

Figura 5. Efeito da dieta nos níveis de colesterol hepático $\left(\mathrm{mg}^{\mathrm{d}} \mathrm{dL}^{-1}\right)$ em ratos machos Wistar Hipercolesterolêmicos ${ }^{\star}$, durante 30 e 60 dias de ensaio biológico. $\left(^{*}\right)$ valores médios de 6 animais, onde: T0 = valor inicial; dieta $1=$ dieta $15 \%$ caseína; dieta $2=5 \%$ de farinha de abacate; dieta $3=15 \%$ de farinha de abacate; dieta $4=25 \%$ de farinha de abacate; e as letras diferentes mostram diferença estatística significativa ( $<<0,05)$.
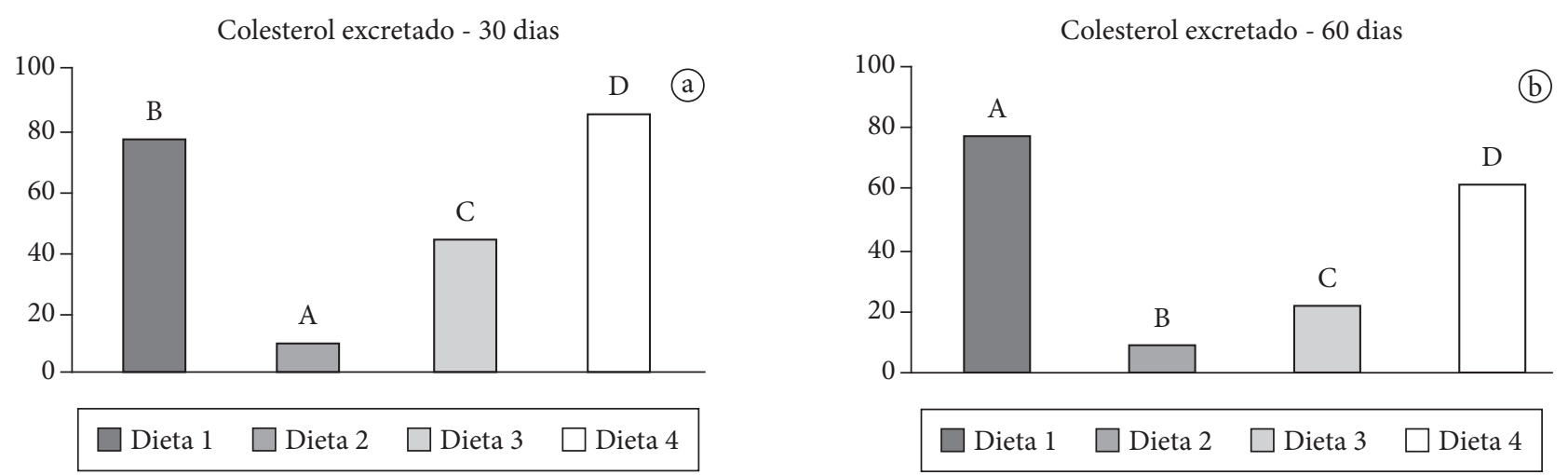

Figura 6. Efeito da dieta nos níveis de colesterol excretado $\left(\mathrm{mg}^{\mathrm{d}} \mathrm{dL}^{-1}\right)$ em ratos machos Wistar Hipercolesterolêmicos ${ }^{\star}$, durante 30 e 60 dias de ensaio biológico. $\left({ }^{*}\right)$ valores médios de 6 animais, onde: T0 = valor inicial; dieta $1=$ dieta $15 \%$ caseína; dieta $2=5 \%$ de farinha de abacate; dieta $3=15 \%$ de farinha de abacate; dieta $4=25 \%$ de farinha de abacate; e as letras diferentes mostram diferença estatística significativa $(\mathrm{p}<0,05)$.

O efeito hipocolesterolêmico dos AG das famílias ômega 3 e ômega 6 é conseqüência da modificação das membranas celulares e das lipoproteínas, do aumento da excreção biliar e fecal do colesterol, além da redução na síntese do VLDL no fígado (BRITISH NUTRITION FOUNDATION, 1992).

No organismo, os fitosteróis, dos quais os mais comuns são o campesterol, o sitosterol e o estigmasterol, atuam na diminuição da absorção de colesterol no intestino delgado por um mecanismo de competição, com conseqüente aumento na excreção fecal. Esta competição ocorre porque a estrutura química dos fitosteróis é semelhante à do colesterol, diferindo no tamanho da cadeia (PIIRONEN et al., 2000; NESTEL et al., 2001).

De acordo com Normén et al. (2000), devido à baixa absorção intestinal, os fitosteróis deslocam o colesterol para fora da micela na luz intestinal, bloqueando parcialmente sua absorção, ou seja, reduzem a capacidade de transporte de colesterol pela micela. Portanto, a ingestão de alimentos contendo ésteres de fitosterol reduz a absorção do colesterol dietético. O colesterol não absorvido é eliminado nas fezes juntamente com os fitosteróis, que são muito pouco absorvidos.
O mecanismo de ação dos fitosteróis ocorre da seguinte maneira: para que o colesterol se torne solúvel, há necessidade de ser incorporado às micelas, no intestino, caso contrário, permanecerá insolúvel e será eliminado nas fezes. Quando os fitosteróis estão presentes na dieta, são quebrados em esteróis livres e ácidos graxos, sendo assim introduzidos nas micelas, impedindo a entrada ou o deslocamento do colesterol para elas. Devido à baixa absorção intestinal, os fitosteróis deslocam o colesterol para fora da micela na luz intestinal, bloqueando parcialmente sua absorção, ou seja, reduzem a capacidade de transporte de colesterol pela micela (NORMÉN, 2000). Portanto, a ingestão de alimentos contendo ésteres de fitosterol, como o abacate, reduz a absorção do colesterol dietético. O colesterol não absorvido é eliminado nas fezes juntamente com os fitosteróis, que são muito pouco absorvidos (WESTSTRATE; MEIJER, 1998).

\section{Conclusões}

A suplementação da dieta com farinha de abacate reduziu os níveis de colesterol sanguíneo, hepático e LDL, aumentou o colesterol excretado e manteve os níveis de HDL e triglicérides 
em ratos hipercolesterolêmicos. Isso demonstra que a dieta tem papel fundamental na redução dos níveis de colesterol.

\section{Referências bibliográficas}

ASP, N. G.; JOHANSSON, C. G.; HALLMER, H. Rapid enzymatic assay of insoluble and soluble dietary fiber. Journal of Agricultural Food Chemistry, v. 31, n. 3, p. 476-482, May 1983.

AOAC - ASSOCIATION OFFICIAL ANALYTICAL CHEMISTS. Official methods of analysis. 16 ed. Washington, 1995.

BRITISH NUTRITION FOUNDATION. Unsaturated fatty acids: nutritional and physiological significance. London: Chapman \& Hall, 1992.

DUESTER, K. C. Avocado fruit is a rich source of beta-sitosterol. Journal of the American Dietetic Association, v. 101, n. 4, p. 404-405, 2001.

FRIEDEWALD, W. T.; LEVY, R. I.; FREDRICKSON, D. S. Estimation of the concentration of low-density lipoprotein cholesterol in plasma, without use of the preparative ultracentrifuge. Clinical Chemistry, v. 18 , n. 6, p. 499-502, 1972.

HALLIKAINEN, M. A. et al. Comparison of the effects of plantsterol ester and plant stanol ester-enriched margarine in lowering serum cholesterol concentrations in hypercholesterolaemic subjects on a low-fat diet. European Journal of Clinical Nutrition, v. 54, p. 715-725, 2000.

HAUG, A.; HOSTMARK, A. T. Lipoprotein lipases, lipoproteins and tissue lipids in rats fed fish oil or coconut oil. The Journal of Nutrition, v. 117, n. 6, p. 1011-1017, 1987.

HENDRIKS, H. F. J. Spreads enriched with three differents levels of vegetables oil sterols and the degree of cholesterol lowering in normocholesterolemic and midly hypercholesterolemic subjects. European Journal of Clinical Nutrition, v. 53, n. 4, p. 319-327, 1999.

HU, F. B. Plant-based foods and prevention of cardiovascular disease: an overview. The American Journal of Clinical Nutrition, v. 78, n. 3, p. 544-551, 2003.

JUDD, J. T. et al. Plant sterol esters lower plasma lipids and most carotenoids in mildly hypercholesterolemic adults. Lipids, v. 37, n. 1, p. 33-34, 2002.

LOTTENBERG, A. M. P. et al. Eficiência dos ésteres de fitoesteróis alimentares na redução dos lípides plasmáticos em hipercolesterolêmicos moderados. Arquivos Brasileiros de Cardiologia, v. 79, n. 2, p. 139-142, 2002.

MAGALHÃES, C. C.; CHAGAS, A. C. P.; Da LUZ, P. L. Importância do HDL-colesterol como preditor de risco para eventos cardiovasculares. Revista da Sociedade de Cardiologia do Estado de São Paulo, v. 12, n. 4, p. 560-568, 2002.

MAZUR, W. M.; DUKE, J. A.; WAHALA, K. Isoflavonoids and lignans in legumes: nutritional and health aspects in humans. The Journal of Nutritional Biochemistry, v. 9, n. 4, p. 193-200, 1998.

MCGOWAN, M. P. Am I at risk for developing heart disease? Heart fitness for life. New York: Oxford, 1997.

MOGHANDASIAN, M. H.; FROHLICH, J. J. Effects of dietary phitosterols on cholesterol metabolism and atherosclerosis: Clinical and Experimental Evidence. American Journal of Medicine, p. 107, 1999.

NAVEH, E. et al. Defatted Avocado Pulp Reduces Body Weight and Total Hepatic Fat But Increases Plasma Cholesterol in Male Rats Fed Diets with Cholesterol. The American Society for Nutritional Sciences, v. 132, p. 2015-2018, 2002.

NEIL, H. A. W.; MEIJER, G. W.; ROE, L. S. Randomised controlled trial of use by hypercholesterolaemic patients of vegetable oil sterol-enriched fat spread. Atherosclerosis, v. 156, p. 329-337, 2001.

NESTEL, P. et al. Cholesterol-lowering effects of plant sterol esters and non-esterified stanols in margarine, butter and low-fat foods. European Journal of Clinical Nutrition, v. 55, n. 12, p. 1084-1090, 2001.

NORMÉN, L. Soy sterol esters and $\beta$-sitostanol ester as inhibitors of cholesterol absorption in human small bowel. American Journal Clinical Nutrition, v. 71, p. 908-913, 2000.

O'KEEFE, J. H.; NELSON, J.; HARRIS, W. B. Life-style change for coronary artery disease. Postgraduate Medicine, v. 99, n. 2, p. 89-106, 1996.

PIIRONEN, V. et al. Review - Plant sterols: biosynthesis, biological function and their importance to human nutrition. Journal of Sciences Food and Agriculture, v. 80, n. 7, p. 939-966, 2000.

PREGNOlatTO, W.; PREGNOLATTO, N. P. (Coord.). Normas analíticas do Instituto Adolfo Lutz. 3 ed. São Paulo: Instituto Adolfo Lutz, 1985.

REBOLLO, A. J. G. et al. Effects of comsumption of meat product rich in monounsaturated fatty acids (the ham from the Iberian pig) on plasma lipids. Nutrition Research, v. 18, n. 12, p. 743-750, 1998.

REEVES, P. G.; NIELSEN, F. H.; FAHEY, G. C. AIN-93: purified diets for laboratory rodents: final report of the american institute of nutrition ad hoc writing committee on the reformulation of the AIN-76A Rodent Diet. The Journal of Nutrition, v. 123, n. 11, p.1939-1951, 1993.

RIQUE, A. B. R.; SOARES, E. A.; MEIRELLES, C. M. Nutrição e exercício na prevenção e controle das doenças cardiovasculares. Revista Brasileira de Medicina do Esporte, v. 8, n. 6, p. 244-254, 2002.

SANTOS, R. D. (Coord.). III Diretrizes brasileiras sobre dislipidemias e diretriz de prevenção da aterosclerose do Departamento de Aterosclerose da Sociedade Brasileira de Cardiologia. Arquivos Brasileiros de Cardiologia, v. 77, n. 1, p. 1-48, 2001.

SANTOS, R. D. et al. III Diretrizes brasileiras sobre dislipidemias e diretrizes de prevenção da aterosclerose do Departamento de Aterosclerose da Sociedade Brasileira de Cardiologia. Arquivos Brasileiros de Cardiologia, v. 77, n. 1, p. 1-191, 2001.

SAS Institute. SAS user's guide: statistic. Version 6. 12 ed. Cary: SAS Institute, 1998.

TANGO, J. S.; CARVALHO, C. R. L.; SOARES, N. B. Caracterização Física e química de frutos de abacate visando a seu potencial para extração de óleo. Revista Brasileira de Fruticultura, v. 26, n. 1, p. 17-23, 2004.

TURATTI, J. M. et al. Caracterização do óleo de abacate obtido por diferentes processos de extração. Boletim do ITAL, v. 22, p. 267-284, 1985.

VALENZUELA, B. A.; GARRIDO, G. A. Os fitoesteróis: agentes hipocolesterolêmicos naturais de origem não farmacológica. Revista Chile Nutrition, v. 27, n. 2, p. 220-225, 2000.

WESTSTRATE, J. A.; MEIJER, G. W. Plant sterol-enriched margarines and reduction of plasma total- and LDL-cholesterol concentrations in normocholesterolaemic and mildly hypercholesterolaemic subjects. European Journal of Clinical Nutrition, v. 52, n. 5, p. 334-343, 1998. 